\title{
Real-world Antihypertensive Treatment Patterns, Treatment Adherence, and Blood Pressure Control in the Elderly: An Italian Awareness-raising Campaign on Hypertension by Senior Italia FederAnziani, the Italian Society of Hypertension and the Italian Federation of General Practitioners
}

\author{
Rita Del Pinto ${ }^{1}$. Giovambattista Desideri ${ }^{2} \cdot$ Claudio Ferri $^{1} \cdot$ Enrico Agabiti Rosei $^{3,4}$
}

Received: 4 May 2021 / Accepted: 13 June 2021 / Published online: 29 June 2021

(c) The Author(s) 2021

\begin{abstract}
Introduction Achieving hypertension control is beneficial regardless of age. Fixed-combination pills have the potential of increasing adherence to treatment, improving the benefit/risk ratio, and simplifying therapy, with resulting convenience especially in the elderly.

Aim We examined real-world antihypertensive treatment adherence and hypertension control rates in a cohort of Italian elderly individuals, enrolled in a prospective, pragmatic awareness-raising campaign on blood pressure (BP).

Methods 13196 treated hypertensive elderly (mean age 73.2 \pm 7.5 years, $55.5 \%$ women) were recruited through opportunistic sampling, answered a brief questionnaire on antihypertensive therapy, and were followed-up for 6 months, when BP was measured as per routine care. Controlled hypertension was defined as BP $<140 / 90 \mathrm{mmHg}$. Real-world treatment adherence and hypertension control rates were evaluated at 6 months according to different treatment patterns (fixed-dose versus free combinations), using Yates correction for continuity to assess likelihood estimates for differences between treatments.

Results 10551 participants (80\%) were on a single-pill therapy and 3445 were on a fixed combination therapy of two (24.8\%) or three (1.3\%) drugs. Individuals on a fixed combination therapy were more adherent to treatment than the counterparts $(\mathrm{p}<0.001)$. Full adherence increased with the number of drugs/pill among single-pill users $(47.5 \%, 68.5 \%$, and $100 \%$ with 1, 2, or 3 drugs/pill; $p<0.001)$. Hypertension control rates were $70 \%$ and $65.2 \%(p=0.001)$ according to fixed or free combinations of two drugs and $71 \%$ and $63.9 \%(\mathrm{p}=0.321)$ for fixed or free combinations of three drugs.

Conclusions Real-world data suggest that simplified treatment strategies and use of fixed combinations improve adherence to antihypertensive therapy and BP control in the elderly.
\end{abstract}

Keywords Real-world analysis $\cdot$ Elderly $\cdot$ Adherence to therapy $\cdot$ Hypertension control $\cdot$ Fixed-dose combinations

Enrico Agabiti Rosei

enrico.agabitirosei@unibs.it

1 Department of Life, Health and Environmental Sciences, Division of Internal Medicine and Nephrology, Hypertension and Cardiovascular Prevention Unit, University of L'Aquila, San Salvatore Hospital, L'Aquila, Italy

2 Department of Life, Health and Environmental Sciences, Division of Geriatrics, University of L'Aquila, SS. Filippo and Nicola Hospital, Avezzano, AQ, Italy

3 Department of Clinical and Experimental Sciences, Clinica Medica Generale, University of Brescia, P.le Spedali Civili, 1, 25123 Brescia, Italy

4 Department of Medicine, Azienda Spedali Civili di Brescia, Brescia, Italy

\section{Introduction}

Hypertension affects $30-45 \%$ of individuals globally, and becomes progressively more common with advancing age, with a prevalence of $60-75 \%$ in people aged 60 years and older [1]. Based on clinical trials data, the benefits of achieving hypertension control translate into a substantial reduction in cardiovascular morbidity and death, as well as in global mortality, even in the very old [2-4]. Population data from individuals aged 85 years or older, including frail ones, also indicate that a good adherence to antihypertensive treatment is associated with a reduced risk of cardiovascular events and mortality [5]. Thus, current guidelines on the 
management of hypertension agree in recommending that high blood pressure (BP) should be properly treated even at the most advanced ages [6, 7].

Observational data, however, indicate that hypertension control rates remain largely unsatisfactory among treated individuals, with less than $40 \%$ reaching a target $\mathrm{BP}$ of $<140 / 90 \mathrm{mmHg}[1,8]$. When treating the elderly, physicians might be concerned regarding the safety of BP lowering, the risk of adverse effects related to antihypertensive medications, and that of drug interactions in the presence of treated comorbidities [4, 9, 10]. In parallel, older individuals are often prescribed a complex treatment regimen comprising multiple medications and repeated dosings for comorbid conditions, which accounts for reduced compliance $[4,10,11]$. In this context, fixed-combination treatment strategies have the advantages of being effective with the use of lower dosages, improving the benefit/risk ratio, simplifying therapy, and increasing adherence to treatment [12-14], thereby representing an option in the management of hypertension in the elderly [15]. Fixed-combination treatment strategies were also reported to have the potential of increasing BP control rates to 70-80\% [16, 17], which might translate into significant benefit on major cardiovascular events and mortality.

The aim of this study was to determine real-world antihypertensive treatment adherence and hypertension control rates in a cohort of Italian elderly individuals according to the observed patterns of antihypertensive medications use.

\section{Methods}

\subsection{Study Type}

We analyzed a prospective, nationwide, pragmatic awareness-raising campaign on BP among treated hypertensive individuals aged $\geq 65$ years, conducted between January 01 , 2019 and December 31, 2019 with the organizing and scientific support of Senior Italia FederAnziani, the Italian Society of Hypertension and the Italian Federation of General Practitioners. The campaign aimed at evaluating real-world antihypertensive treatment adherence and BP control rates in relation to different treatment patterns in the elderly. It was promoted nationally through advertisements and locally by means of promotional material at the screening sites, which included the general practitioner's office, hypertension units, hospitals, and primary care clinics. Volunteers were recruited at local sites using convenience sampling. Having been made aware of the campaign, individuals who wished to participate were self-referred. Individuals who depended on caregivers for the management of therapy were excluded from participation. Follow-up was 6 months from the enrollment date (index date).

The campaign fulfilled the principles stated in the Declaration of Helsinki, it was entirely observational, and ethics approval was not required. Participation was completely voluntary, and signed informed consent was obtained from each participant at enrollment. Consent to participation included permission to use aggregated, anonymized data for scientific divulgation purposes. Data were de-identified at enrollment, and identification of participants was therefore not possible.

\subsection{Study Outcomes}

The study outcomes were real-world antihypertensive treatment adherence and BP control rates in treated hypertensive elderly individuals in relation to different antihypertensive treatment patterns (monotherapy, fixed-dose double or triple combination therapy, and free combinations of 2 or 3 drugs).

\subsection{BP Measurement and Control}

Given the pragmatic study design, the frequency of BP measurements from the index date to the end of follow-up could vary among participants, reflecting methods already adopted in previous surveys on BP [18, 19]. For the same reason, locally available devices were used to measure BP with a recommendation to use validated automated devices where possible [20]. Standard methods for BP measurement were recommended to include three sitting recordings taken at 1 minute intervals, from either the right or left arm. The mean of the second and third readings was recorded and used for the analyses; otherwise, when only one or two measurements were available, the single reading or the second reading were recorded, respectively [20].

Controlled hypertension was defined as a systolic BP $<140 \mathrm{mmHg}$ and a diastolic BP < $90 \mathrm{mmHg}$ [15].

\subsection{Antihypertensive Treatment Adherence}

Antihypertensive treatment adherence was assessed using a simplified interval-based approach (months covered/ months in the interval) [16], and defined as the ratio between the number of pills in boxes used and those expected in 6 months. Rounding to the nearest integer was performed. A participant was defined as fully adherent when the adherence rate was $\geq 80 \%$ [17].

\subsection{Additional Data}

Along with BP measurements, limited data (age, gender, number and type of antihypertensive medications, hospitalizations in the previous year) were collected anonymously from each participant and recorded on a centralized database 
according to previous literature [20]. Information on antihypertensive medications included their class, the number of pills taken on a daily basis, whether the participant was on fixed-dose or free combinations, and the number of active ingredients per pill in case of fixed-combination therapy.

\subsection{Statistical Analysis}

Descriptive analysis of participants' characteristics was assessed with unpaired $t$ tests for continuous variables (mean \pm standard deviation, SD) and $\chi^{2}$ tests for categorical variables (N, \%).

BP was treated as both a numeric value $(\mathrm{mmHg})$ and a categorical variable (controlled or uncontrolled hypertension). Similarly, adherence was described in terms of a continuous (mean \pm standard deviation) and a dichotomous variable $(<80 \% ; \geq 80 \%)$.

Treatment adherence and hypertension control were assessed based on the number of antihypertensive pills (1, 2 , or 3$)$, the number of active ingredients per pill $(1,2$, or 3$)$, and being on a fixed-dose double or triple combination or a free combination of 2 or 3 active ingredients.

The likelihood estimate for the difference in hypertension control rates (95\% confidence intervals) [18] according to being on a fixed-dose or a free combination therapy was assessed using Yates correction for continuity.

A subgroup analysis on individuals with pulse pressure (PP) $\geq 60 \mathrm{mmHg}$ was performed where treatment adherence and BP control were assessed based on treatment strategy (monotherapy, fixed-dose double or triple combination, and free combination of 2 or 3 active ingredients).

Data were analyzed as recorded, without imputation for missing data.

Analyses were conducted using R (v 4.0.2) [24]. Statistical significance was set at $\mathrm{p}<0.05$.

\section{Results}

A total of 13,196 treated hypertensive elderly individuals (mean age $73.2 \pm 7.5$ years, $55.5 \%$ women) participated in the campaign. 10,551 individuals $(80 \%)$ were on a single antihypertensive pill, 2500 (18.9\%) were on two pills, and the remaining $145(1.1 \%)$ were on three antihypertensive pills. None of them were on a four-pill antihypertensive therapy. 3272 individuals ( $24.8 \%$ of total) were on fixed-dose double combination therapy; of them, 54 (1.6\%) were taking two fixed-dose double combination pills. 173 individuals $(1.3 \%$ of total) were on a fixed-dose triple combination therapy; of them, 11 (6.3\%) were concomitantly taking one or two additional antihypertensive pills, either single drug or fixed-dose double combination.
Only 5721 individuals (43.3\%) were fully adherent to therapy (adherence rate $\geq 80 \%$ ). Globally, 9027 participants (68.4\%) had controlled hypertension (Table 1). Compared with participants with uncontrolled hypertension, they tended to be women on less antihypertensive pills $(1.20 \pm 0.43$ versus $1.23 \pm 0.44, \mathrm{p}=0.006)$, with greater treatment adherence $(47.9 \%$ versus $33.5 \% ; \mathrm{p}<0.001)$, and $24.4 / 7 \mathrm{mmHg}$ lower systolic/diastolic BP $(\mathrm{p}<0.001)$. They more often reported hospitalizations during the previous year. No difference in mean age was reported according to BP control. Similarly, no difference in the use of fixed-dose combinations nor in the number of active ingredients was recorded between the two groups. The use of beta-blockers and diuretics was similar between the two groups (Table 1).

Compared with men, women tended to be slightly younger $(73.1 \pm 7.3$ versus $73.4 \pm 7.6$ years, $p=0.021)$, had lower prevalence of hospitalizations in the previous year $(15.5 \%$ versus $17.7 \%, p<0.001)$, and underwent less $\mathrm{BP}$ measurements during the campaign $(32.5 \pm 20.6$ versus $33.50 \pm 21.7, \mathrm{p}=0.005)$. Women were also more adherent to antihypertensive therapy ( $45.1 \%$ versus $41.2 \%, \mathrm{p}<0.001$ ) and more likely to have controlled hypertension $(70.1 \%$ versus $66.3 \%, \mathrm{p}<0.001)$ than men. No difference in the number of daily pills was observed based on sex $(1.21 \pm 0.43)$ versus $1.21 \pm 0.44, \mathrm{p}=0.838$ ).

\subsection{Real-world Treatment Adherence Based on Medications Patterns}

\subsubsection{Number of Antihypertensive Pills}

Fully adherent individuals (treatment adherence rate $\geq 80 \%$ ) were $53.5 \%, 3.1 \%$, and $0 \%$ of those on one, two, and three antihypertensive pills, respectively ( $p<0.001)$ (Table 2). Individuals on a single antihypertensive pill had a mean adherence rate of $73.4 \pm 15.7 \%$, while those on two or three pills had a mean adherence rate of $52.9 \pm 11.6 \%$ and $38 \pm 7.8 \%$, respectively $(\mathrm{p}<0.001)$ (Table 2$)$.

Interestingly, full adherence progressively improved with increasing the number of active ingredients per pill among individuals taking a single pill (from $47.5 \%$ to $68.5 \%$ to $100 \%$ in the presence of 1,2 , or 3 active ingredients; $\mathrm{p}<0.001$ ) (Fig. 1, panel A). The same progression was observed in terms of mean adherence to therapy (from $71.5 \pm 15.7 \%$ to $78.3 \pm 14.3 \%$ to $91.4 \pm 14.5 \%$; $<0.001$ ) (Fig. 1, panel B).

\subsubsection{Fixed-dose Combinations Versus Free Combinations}

Treatment adherence according to being on a fixed-dose double combination or a free combination of two pills is reported in Table 3. Fully adherent individuals (treatment adherence rate $\geq 80 \%$ ) were $68.5 \%$ and $1.9 \%$ of those on 
Table 1 Demographic and clinical characteristics of the treated hypertensive elderly participants enrolled in the campaign $(\mathrm{N}=13196)$, stratified according to BP control

\begin{tabular}{|c|c|c|c|}
\hline Variables & $\begin{array}{l}\text { Uncontrolled } \\
\text { hypertension }\end{array}$ & Controlled hypertension & $P$ value \\
\hline $\mathrm{N}$. & 4169 & 9027 & \\
\hline Age (mean (SD)) & $73.11(7.21)$ & $73.25(7.58)$ & 0.293 \\
\hline Men $(\%)$ & $1976(47.4)$ & $3894(43.1)$ & $<0.001$ \\
\hline Hospitalized in the past year (\%) & $618(14.8)$ & $1559(17.3)$ & $<0.001$ \\
\hline Adherence (mean (SD)) & $66.00(15.82)$ & $70.63(17.76)$ & $<0.001$ \\
\hline Fully adherent participants (\%) & $1398(33.5)$ & $4323(47.9)$ & $<0.001$ \\
\hline BP measurements (mean (SD)) & $30.09(18.74)$ & $34.23(21.99)$ & $<0.001$ \\
\hline Systolic BP, mmHg (mean (SD)) & $149.19(8.47)$ & $124.80(8.89)$ & $<0.001$ \\
\hline Diastolic BP, mmHg (mean (SD)) & $80.78(7.60)$ & $73.79(7.39)$ & $<0.001$ \\
\hline $\mathrm{PP}, \mathrm{mmHg}($ mean $(\mathrm{SD}))$ & $68.42(10.41)$ & $51.02(9.12)$ & $<0.001$ \\
\hline Antihypertensive pills (mean (SD)) & $1.23(0.44)$ & $1.20(0.43)$ & 0.006 \\
\hline Active ingredients in therapy (mean (SD)) & $1.49(0.65)$ & $1.49(0.66)$ & 0.743 \\
\hline No fixed combinations $(\%)$ & $3124(74.9)$ & $6627(73.4)$ & 0.068 \\
\hline Fixed-dose double combinations (\%) & & & 0.098 \\
\hline None & $3174(76.1)$ & $6747(74.7)$ & \\
\hline 1 & $983(23.6)$ & $2238(24.8)$ & \\
\hline 2 & $12(0.3)$ & $42(0.5)$ & \\
\hline Fixed-dose triple combination (\%) & $51(1.2)$ & $122(1.4)$ & 0.603 \\
\hline ACE inhibitors (\%) & & & 0.025 \\
\hline 0 & $2822(67.7)$ & $6035(66.9)$ & \\
\hline 1 & $1275(30.6)$ & $2888(32.0)$ & \\
\hline 2 & $70(1.7)$ & $102(1.1)$ & \\
\hline 3 & $2(0.0)$ & $2(0.0)$ & \\
\hline Beta blockers (\%) & & & 0.878 \\
\hline 0 & $3159(75.8)$ & $6808(75.4)$ & \\
\hline 1 & $968(23.2)$ & $2121(23.5)$ & \\
\hline 2 & $41(1.0)$ & $97(1.1)$ & \\
\hline 3 & $1(0.0)$ & $1(0.0)$ & \\
\hline Calcium channel blockers (\%) & & & 0.007 \\
\hline 0 & $3075(73.8)$ & $6642(73.6)$ & \\
\hline 1 & $1041(25.0)$ & $2319(25.7)$ & \\
\hline 2 & $53(1.3)$ & $66(0.7)$ & \\
\hline Diuretics (\%) & & & 0.124 \\
\hline 0 & $2925(70.2)$ & $6274(69.5)$ & \\
\hline 1 & $1160(27.8)$ & $2510(27.8)$ & \\
\hline 2 & $84(2.0)$ & $242(2.7)$ & \\
\hline 3 & $0(0.0)$ & $1(0.0)$ & \\
\hline Angiotensin II receptor blockers (\%) & & & 0.025 \\
\hline 0 & $2916(69.9)$ & $6507(72.1)$ & \\
\hline 1 & $1232(29.6)$ & $2466(27.3)$ & \\
\hline 2 & $21(0.5)$ & $54(0.6)$ & \\
\hline
\end{tabular}

$B P$ blood pressure, $P P$ pulse pressure, $A C E$ angiotensin converting enzyme fixed and free combinations, respectively $(\mathrm{p}<0.001)$ (Table 3). Individuals on a fixed-dose double combination had a mean adherence rate of $78.3 \pm 14.3 \%$, while those on a free combination of two pills had a mean adherence rate of $52.5 \pm 11.7 \%(\mathrm{p}<0.001)($ Table 3$)$.
Treatment adherence according to being on a fixeddose triple combination or a free combination of three pills is reported in Table $4.100 \%$ of individuals on fixed combination therapy were adherent, while the totality of those on free combinations of three pills were nonadherent $(\mathrm{p}<0.001)$ (Table 4$)$. The mean adherence rate 
Table 2 Real-world treatment adherence based on the number of antihypertensive pills

\begin{tabular}{|c|c|c|c|c|}
\hline Variables & 1 pill & 2 pills & 3 pills & $P$ value \\
\hline $\mathrm{N}$. & 10551 & 2500 & 145 & \\
\hline Age (mean (SD)) & $73.14(7.58)$ & $73.31(7.06)$ & $76.40(4.76)$ & $<0.001$ \\
\hline Men $(\%)$ & $4721(44.7)$ & $1062(42.5)$ & $87(60.0)$ & $<0.001$ \\
\hline Hospitalized in the past year (\%) & $1695(16.1)$ & $458(18.3)$ & $24(16.6)$ & 0.039 \\
\hline Adherence (mean (SD)) & $73.44(15.75)$ & $52.95(11.61)$ & $38.04(7.79)$ & $<0.001$ \\
\hline Fully adherent participants (\%) & $5643(53.5)$ & $78(3.1)$ & $0(0.0)$ & $<0.001$ \\
\hline BP measurements (mean (SD)) & $31.97(20.41)$ & $36.76(23.55)$ & $36.28(18.36)$ & $<0.001$ \\
\hline Systolic BP, mmHg (mean (SD)) & $132.15(14.30)$ & $134.00(14.31)$ & $132.52(14.98)$ & $<0.001$ \\
\hline Diastolic BP, mmHg (mean (SD)) & $75.95(8.12)$ & $76.18(8.15)$ & $76.21(8.56)$ & 0.438 \\
\hline PP, mmHg (mean (SD)) & $56.21(12.53)$ & $57.83(12.33)$ & $56.30(13.42)$ & $<0.001$ \\
\hline Controlled hypertension (\%) & $7284(69.0)$ & $1641(65.6)$ & $102(70.3)$ & 0.004 \\
\hline No fixed combinations (\%) & 7795 (73.9) & $1870(74.8)$ & $86(59.3)$ & $<0.001$ \\
\hline Fixed-dose double combinations (\%) & & & & $<0.001$ \\
\hline None & $7957(75.4)$ & $1878(75.1)$ & $86(59.3)$ & \\
\hline 1 & $2594(24.6)$ & $578(23.1)$ & $49(33.8)$ & \\
\hline 2 & $0(0.0)$ & $44(1.8)$ & $10(6.9)$ & \\
\hline Fixed-dose triple combination (\%) & $162(1.5)$ & $10(0.4)$ & $1(0.7)$ & $<0.001$ \\
\hline Active ingredients in therapy (mean (SD)) & $1.28(0.48)$ & $2.27(0.50)$ & $3.49(0.66)$ & $<0.001$ \\
\hline ACE inhibitors (\%) & & & & $<0.001$ \\
\hline 0 & $7313(69.3)$ & $1494(59.8)$ & $50(34.5)$ & \\
\hline 1 & $3238(30.7)$ & $863(34.5)$ & $62(42.8)$ & \\
\hline 2 & $0(0.0)$ & $143(5.7)$ & $29(20.0)$ & \\
\hline 3 & $0(0.0)$ & $0(0.0)$ & $4(2.8)$ & \\
\hline Beta blockers (\%) & & & & $<0.001$ \\
\hline 0 & $8345(79.1)$ & $1577(63.1)$ & $45(31.0)$ & \\
\hline 1 & $2206(20.9)$ & $822(32.9)$ & $61(42.1)$ & \\
\hline 2 & $0(0.0)$ & $101(4.0)$ & $37(25.5)$ & \\
\hline 3 & $0(0.0)$ & $0(0.0)$ & $2(1.4)$ & \\
\hline Calcium channel blockers (\%) & & & & $<0.001$ \\
\hline 0 & $8090(76.7)$ & $1541(61.6)$ & $86(59.3)$ & \\
\hline 1 & $2461(23.3)$ & $847(33.9)$ & $52(35.9)$ & \\
\hline 2 & $0(0.0)$ & $112(4.5)$ & $7(4.8)$ & \\
\hline Diuretics (\%) & & & & $<0.001$ \\
\hline 0 & $7951(75.4)$ & $1186(47.4)$ & $62(42.8)$ & \\
\hline 1 & $2511(23.8)$ & $1097(43.9)$ & $62(42.8)$ & \\
\hline 2 & $89(0.8)$ & $217(8.7)$ & $20(13.8)$ & \\
\hline 3 & $0(0.0)$ & $0(0.0)$ & $1(0.7)$ & \\
\hline Angiotensin II receptor blockers (\%) & & & & $<0.001$ \\
\hline 0 & $7676(72.8)$ & $1654(66.2)$ & $93(64.1)$ & \\
\hline 1 & $2875(27.2)$ & $781(31.2)$ & $42(29.0)$ & \\
\hline 2 & $0(0.0)$ & $65(2.6)$ & $10(6.9)$ & \\
\hline
\end{tabular}

$B P$ blood pressure, $P P$ pulse pressure, $A C E$ angiotensin converting enzyme was $91.4 \pm 8.5 \%$ among individuals on a fixed-dose triple combination, and of $39.3 \pm 8.3 \%$ among those on a free combination of three pills $(\mathrm{p}<0.001)$ (Table 4$)$.

\subsubsection{Real-world Hypertension Control Rates According to Fixed-dose or Free Combinations}

Among participants on a treatment strategy based on two active ingredients ( $\mathrm{N}=4472), 2594$ (58\%; mean age $72.7 \pm 7.2$ years; $56.2 \%$ women) were on a fixed-dose combination (Table 3). Based on a mean of $33.1 \pm 20.5 \mathrm{BP}$ 
Fig. 1 Full adherence rates (A) and mean adherence to therapy (B) in treated hypertensive elderly individuals taking a single pill containing 1,2 , or 3 active ingredients. Fully adherent individuals (adherence $\geq 80 \%$ ) were $47.5 \%, 68.5 \%$ and $100 \%$ ( $\mathrm{p}<0.001$ for multiple comparisons), respectively. Mean adherence to therapy increased from $71.5 \pm 15.7 \%$ to $78.3 \pm 14.3 \%$ to $91.4 \pm 14.5 \%$, respectively ( $\mathrm{p}<0.001$ for multiple comparisons). $F C$ fixed-combinations
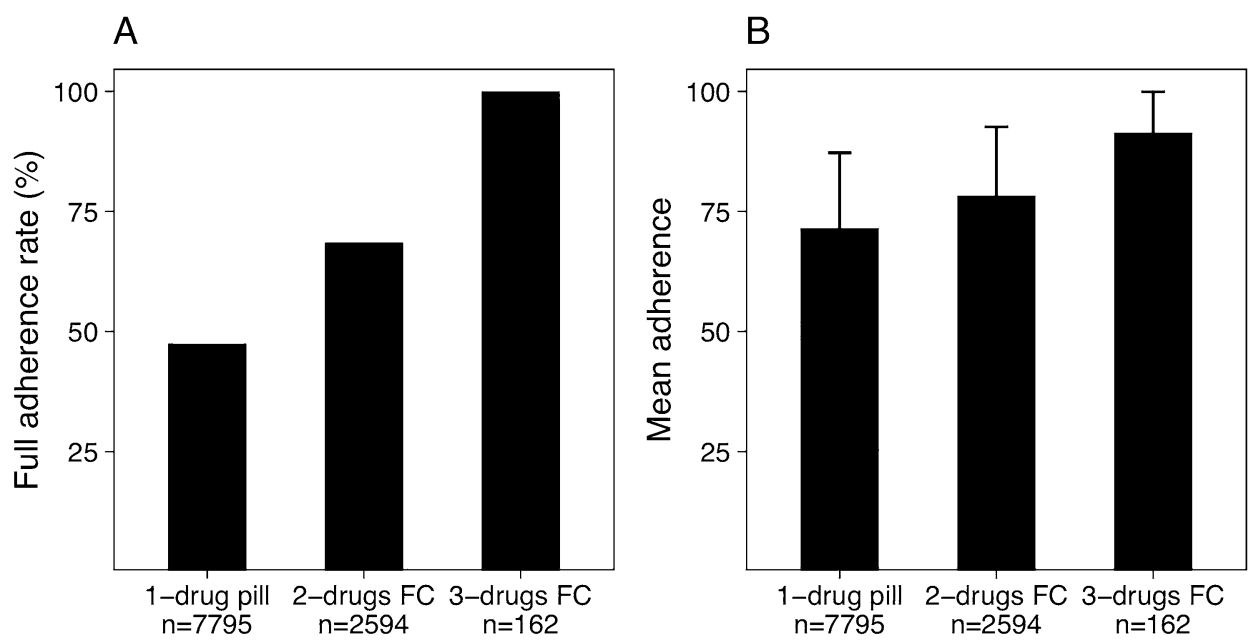

Table 3 Real-world treatment adherence and BP profile based on fixed-dose combinations versus free combinations of two active ingredients

Table 4 Real-world treatment adherence and BP profile based on fixed-dose combinations versus free combinations of three active ingredients

\begin{tabular}{lllr}
\hline & $\begin{array}{l}\text { One fixed-dose double } \\
\text { combination }\end{array}$ & $\begin{array}{l}\text { Free combination of } 2 \\
\text { active ingredients }\end{array}$ & P value \\
\hline $\mathrm{N}$ & 2594 & 1878 & \\
Age (mean (SD)) & $72.72(7.17)$ & $73.28(7.11)$ & 0.009 \\
Men (\%) & $1137(43.8)$ & $795(42.3)$ & 0.333 \\
Adherence (mean (SD)) & $78.26(14.34)$ & $52.49(11.66)$ & $<0.001$ \\
Fully adherent participants (\%) & $1776(68.5)$ & $36(1.9)$ & $<0.001$ \\
BP measurements (mean (SD)) & $33.15(20.54)$ & $35.73(23.10)$ & $<0.001$ \\
Systolic BP, mmHg (mean (SD)) & $131.91(14.09)$ & $133.91(14.54)$ & $<0.001$ \\
Diastolic BP, mmHg (mean (SD)) & $75.70(7.71)$ & $76.36(8.08)$ & 0.006 \\
PP, mmHg (mean (SD)) & $56.23(12.60)$ & $57.57(12.43)$ & $<0.001$ \\
Controlled hypertension (\%) & $1816(70.0)$ & $1224(65.2)$ & 0.001 \\
Antihypertensive pills (mean (SD)) & $1.00(0.00)$ & $2.00(0.00)$ & $<0.001$ \\
\hline
\end{tabular}

$B P$ blood pressure, $P P$ pulse pressure

\begin{tabular}{lllr}
\hline & $\begin{array}{l}\text { Fixed-dose triple com- } \\
\text { bination }\end{array}$ & $\begin{array}{l}\text { Free combination of 3 } \\
\text { active ingredients }\end{array}$ & P value \\
\hline $\mathrm{N}$ & 162 & 86 & \\
Age (mean (SD)) & $71.97(8.30)$ & $75.80(5.23)$ & $<0.001$ \\
Men (\%) & $82(50.6)$ & $58(67.4)$ & 0.016 \\
Adherence (mean (SD)) & $91.40(8.53)$ & $39.33(8.27)$ & $<0.001$ \\
Fully adherent participants (\%) & $162(100.0)$ & $0(0.0)$ & $<0.001$ \\
BP measurements (mean (SD)) & $21.52(5.68)$ & $35.40(18.62)$ & $<0.001$ \\
Systolic BP, mmHg (mean (SD)) & $130.27(12.26)$ & $133.78(16.12)$ & 0.056 \\
Diastolic BP, mmHg (mean (SD)) & $76.95(9.13)$ & $76.08(9.09)$ & 0.475 \\
PP, mmHg (mean (SD)) & $53.30(11.39)$ & $57.70(14.64)$ & 0.010 \\
Controlled hypertension (\%) & $115(71.0)$ & $55(64.0)$ & 0.321 \\
Antihypertensive pills (mean (SD)) & $1.00(0.00)$ & $3.00(0.00)$ & $<0.001$ \\
\hline
\end{tabular}

$B P$ blood pressure, $P P$ pulse pressure 
measurements, their mean systolic BP was $131.9 \pm 14.1$ $\mathrm{mmHg}$ and their mean diastolic $\mathrm{BP}$ was $75.7 \pm 7.7 \mathrm{mmHg}$. Individuals on a double free combination (42\%; mean age $73.3 \pm 7.1$ years; $57.7 \%$ women) had mean systolic BP of $133.9 \pm 14.5 \mathrm{mmHg}$ and mean diastolic BP of $76.4 \pm 8.1$ mmHg based on a mean of $35.7 \pm 23.1$ BP measurements. Individuals taking a fixed-dose double combination had 2 $\mathrm{mmHg}$ lower systolic BP $(\mathrm{p}<0.001)$ and $0.7 \mathrm{mmHg}$ lower diastolic BP $(p=0.015)$ than participants taking a free combination of two active ingredients.

Hypertension control rates varied from $70.0 \%$ (95\% CI 68.2-71.8; $\mathrm{p}<0.001)$ in participants on fixed-dose double combination to $65.2 \%$ (95\% CI 63.0-67.3; p < 0.001) among those on free dose double combination. The likelihood estimate for the difference between groups was $4.8 \%$ (95\% CI $2.0-7.7 ; \mathrm{p}=0.001$ ).

Of participants taking three active ingredients $(\mathrm{N}=248)$, 162 (65.3\%; mean age $72.0 \pm 8.3$ years; $49.4 \%$ women) were on a fixed-dose combination (Table 4). Based on a mean of $21.5 \pm 5.7 \mathrm{BP}$ measurements, their mean systolic BP was $130.3 \pm 12.3 \mathrm{mmHg}$ and their mean diastolic $\mathrm{BP}$ was $76.9 \pm 9.1 \mathrm{mmHg}$. Individuals on a triple free combination (34.7\%; mean age $75.8 \pm 5.2$ years; $32.6 \%$ women) had mean systolic BP of $133.8 \pm 16.1 \mathrm{mmHg}$ and mean diastolic $\mathrm{BP}$ of $76.1 \pm 9.1 \mathrm{mmHg}$ based on a mean of $35.4 \pm 18.6 \mathrm{BP}$ measurements. No significant difference in mean BP values was observed between the groups.

Among participants on fixed-dose triple combination, hypertension control rate was $71.0 \%$ (95\% CI 63.3-77.7; $\mathrm{p}<0.001$ ), whereas it was $63.9 \%$ (95\% CI 52.8-73.8; $\mathrm{p}=0.013)$ among those on free-dose triple combination.
The likelihood estimate for the difference between groups was $0.7 \%$ (95\% CI -6.18 to $20.2 ; \mathrm{p}=0.321$ ).

\subsection{Subgroup Analysis}

The subgroup analysis comprised 4796 individuals with PP $\geq 60 \mathrm{mmHg}$ (mean age $72.8 \pm 7.2,55.7 \%$ women, mean systolic BP $144.9 \pm 11.1 \mathrm{mmHg}$, mean diastolic BP $75.4 \pm 8.5$ $\mathrm{mmHg}$, average number of pills $1.18 \pm 0.40$, average number of active ingredients $1.40 \pm 0.53$ ). Globally, no difference in sex, mean systolic and diastolic BP, and proportion of controlled hypertension was recorded based on the antihypertensive treatment strategy (Table 5). Conversely, mean adherence and the proportion of fully adherent individuals were lower among those on a free combination strategy compared with the fixed combination strategy.

In spite of this, mean systolic BP did not differ between individuals taking 1 fixed-dose double combination and those taking a free combination of 2 active ingredients $(\mathrm{p}=0.128)$.

Conversely, mean systolic BP and mean PP were lower in individuals taking 1 fixed-dose triple combination compared with those on a free combination of 3 active ingredients $(\mathrm{p}<0.0001$ and $\mathrm{p}=0.0018$, respectively), but the proportion of those with controlled hypertension was similar $(\mathrm{p}=0.1831)$. The two groups did not differ in terms of age $(\mathrm{p}=0.0667)$ and $\operatorname{sex}(\mathrm{p}=0.9851)$.

Table 5 Real-world treatment adherence and BP profile based on treatment strategy in the subgroup of participants with PP $\geq 60 \mathrm{mmHg}$ $(\mathrm{N}=4796)$

\begin{tabular}{|c|c|c|c|c|c|c|}
\hline & 1 single active ingredient & $\begin{array}{l}1 \text { fixed-dose } \\
\text { double combi- } \\
\text { nation }\end{array}$ & $\begin{array}{l}1 \text { fixed-dose } \\
\text { triple combina- } \\
\text { tion }\end{array}$ & $\begin{array}{l}\text { Free combination of } \\
2 \text { active ingredients }\end{array}$ & $\begin{array}{l}\text { Free combination of } \\
3 \text { active ingredients }\end{array}$ & $P$ value \\
\hline $\mathrm{n}(\%)$ & $2958(61.7)$ & $966(20.1)$ & $53(1.1)$ & $782(16.3)$ & $37(0.7)$ & \\
\hline Age (mean (SD)) & $72.81(7.26)$ & $71.97(7.21)$ & $73.32(8.46)$ & $73.53(6.84)$ & $76.11(4.11)$ & $<0.001$ \\
\hline Men (\%) & $1325(44.8)$ & $418(43.3)$ & $30(56.6)$ & $333(42.6)$ & $21(56.8)$ & 0.125 \\
\hline $\begin{array}{l}\text { Mean adherence (mean } \\
\text { (SD)) }\end{array}$ & $68.76(15.23)$ & $76.26(14.63)$ & $92.62(8.51)$ & $53.51(11.10)$ & $38.05(7.88)$ & $<0.001$ \\
\hline Full adherence $(\%)$ & $1207(40.8)$ & $613(63.5)$ & $53(100.0)$ & $8(1.0)$ & $0(0.0)$ & $<0.001$ \\
\hline $\begin{array}{l}\text { Systolic BP, mmHg (mean } \\
\text { (SD)) }\end{array}$ & $144.87(11.19)$ & $144.68(10.74)$ & $141.66(13.10)$ & $145.48(11.26)$ & $147.68(9.89)$ & 0.054 \\
\hline $\begin{array}{l}\text { Diastolic BP, mmHg (mean } \\
\quad(\mathrm{SD}) \text { ) }\end{array}$ & $75.38(8.69)$ & $74.93(7.94)$ & $75.34(10.70)$ & $75.93(8.50)$ & $75.89(8.46)$ & 0.198 \\
\hline $\mathrm{PP}, \mathrm{mmHg}($ mean $(\mathrm{SD}))$ & $69.50(7.51)$ & $69.77(7.76)$ & $66.26(6.12)$ & $69.58(8.26)$ & $71.81(10.20)$ & 0.008 \\
\hline $\begin{array}{l}\text { Controlled hypertension } \\
(\%)\end{array}$ & $927(31.3)$ & $322(33.3)$ & $20(37.7)$ & $249(31.8)$ & $9(24.3)$ & 0.534 \\
\hline
\end{tabular}

$B P$ blood pressure, $P P$ pulse pressure 


\section{Discussion}

Real-world data from an Italian nationwide, pragmatic campaign on $\mathrm{BP}$ involving more than 13000 treated hypertensive elderly individuals indicate that the majority $(80 \%)$ were on a single-pill therapy and about one out of 4 were on a fixed combination therapy. Full adherence progressively improved not only with reducing the number of daily pills, but also with increasing the number of active ingredients per pill among individuals taking a single pill. Globally, less than half participants were fully adherent to the prescribed regimen, and about one third did not achieve hypertension control. Individuals with controlled hypertension used less antihypertensive pills and had greater treatment adherence compared with the counterpart.

Our findings are in line with the existing evidence that relates therapeutic complexity with reduced treatment adherence and poor BP control [1], and expand current knowledge on the true impact of real-world treatment strategies on adherence to therapy and BP control in the elderly. Notably, a novel finding of this study is that adherence to therapy among individuals taking a single pill increases with increasing the number of active ingredients in the pill. This observation might reflect increased motivation of patients to comply with treatment, possibly as the effect of therapy simplification or as a consequence of an effective, patient-centered communication strategy. In addition, previous evidence showed that initial combination treatment is associated with a better long-term adherence to the prescribed treatment regimen [12], which might be the case for at least part of the examined cohort. Combination therapy has also the advantage of preventing therapeutic inertia [25], in that the availability of a range of dosages, as well as the possibility to progress from one to two or three drugs whilst remaining on a simple single-pill treatment regimen throughout, translate into improved adherence to therapy and increased BP control [6]. This might contribute to explain the observation of a substantial proportion of the examined cohort achieving BP control despite the suboptimal rate of full adherence.

Reducing the number of daily pills to improve treatment adherence and BP control is particularly relevant when multiple drugs are needed either to control BP or as a result of comorbidities, both of which are common scenarios in the elderly $[4,26]$. We found that participants in this campaign were more adherent and achieved better BP control rates during double fixed combination therapy compared with individuals on the same number of drugs in free combination. Indeed, despite the full adherence to therapy observed in the totality of individuals on a fixed-dose triple combination treatment strategy, this did not translate into a more effective BP control than the counterpart on a 3-drugs freecombination strategy, possibly due to the small sample size.
In fact, the between-groups difference in systolic BP tended to be significant $(-3.5 \mathrm{mmHg}, \mathrm{p}=0.056)$, and the proportion of individuals achieving BP goal in each group (71\% and $63.9 \%, \mathrm{p}=0.321$ ) was similar in magnitude to what was observed in the well larger groups on double combinations treatment strategies, where BP control rates differed significantly based on being on a fixed or free combination strategy (70\% and 65.4\%, respectively; $p=0.001$ ).

Studies suggest that a three-drug combination should control BP in $>80 \%$ of patients, i.e. nearly double the current rate of hypertension control in Europe [16, 17], and about $10 \%$ more than what we observed. It is possible that the observed gap depends on the specific clinical setting that we examined, i.e. elderly individuals. In this context, arterial stiffness and the possible burden of concomitant diseases and related treatments might affect the rate of BP goal achievement $[4,27]$. However, similar findings were described among younger hypertensive individuals (mean age: 56 years) enrolled in The Triple Pill versus Usual Care Management for Patients with Mild-to-Moderate Hypertension (TRIUMPH) trial [28], where the fixed-dose triple combination pill (telmisartan, amlodipine, and chlorthalidone) compared with usual care increased the proportion achieving target BP at 6 months to around $70 \%$ ( $<<0.001$ ), without differences in adherence between groups. Thus, it is also possible that the fixed combination treatment strategy does not allow to exploit chronotherapy to control for specific patterns of hypertension [29]. Also, the possibility of resistant hypertension should be considered when BP remains uncontrolled with three-drug combination therapy, assuming that secondary causes of hypertension and poor adherence to treatment have been excluded, and that the finding has been confirmed by repeated office BP measurement as well as ambulatory and home BP monitoring [6].

Another worth mentioning finding of this real-world analysis is the albeit rare use of multiple concomitant fixed-dose combinations, which is particularly questionable among individuals on a triple-drug fixed-combination treatment strategy. This observation might reflect inaccurate or neglected medication reconciliation, or a lack of active involvement of patients in the healthcare team [30]. As such, it raises relevant issues that need to be addressed for an effective and safe management of the elderly hypertensive patient.

This study has limitations that need to be considered when interpreting the relative findings and their generalizability. The study involved a population of active, relatively fit elderly individuals with mild hypertension, in large part requiring monotherapy. The pragmatic nature of the campaign implied variability in terms of type of BP measurement devices, as well as of inter- and intraindividual frequency of BP measurements. Information on the timing of therapy intake, fasting conditions, as well as on factors that 
might have affected BP values (e.g. cigarette smoking, coffee intake, physical activity, or other health conditions [31-33]) was not available. Adherence to therapy was based on an indirect assessment, and not on the quantification of antihypertensive drugs or their metabolites in urine or blood [34]. However, the campaign involved a large number of treated elderly hypertensive individuals, and depicted a real-world scenario where the actual impact of different treatment strategies on adherence and BP control could be described, which is indeed difficult to replicate in the setting of conventional clinical trials. In addition, some of the reported findings suggest the central role for communication in the healthcare team, both in terms of patients' involvement for raising their awareness and increasing their adherence to therapy, and in terms of medications review and reconciliation.

In conclusion, real-world data from treated elderly hypertensive individuals suggest that simplified treatment strategies and the use of fixed-combination pills improve adherence to therapy and BP control. Patient-centered practice should be applied to provide best care in the usual setting.

Acknowledgements The authors would like to acknowledge Senior Italia, the Italian Society of Hypertension and the Italian Federation of General Practitioners for the organization of the study.

\section{Declarations}

Funding Open access funding provided by Università degli Studi dell'Aquila within the CRUI-CARE Agreement. None specific.

Conflict of interest The Authors declare no conflicts of interests.

Open Access This article is licensed under a Creative Commons Attribution-NonCommercial 4.0 International License, which permits any non-commercial use, sharing, adaptation, distribution and reproduction in any medium or format, as long as you give appropriate credit to the original author(s) and the source, provide a link to the Creative Commons licence, and indicate if changes were made. The images or other third party material in this article are included in the article's Creative Commons licence, unless indicated otherwise in a credit line to the material. If material is not included in the article's Creative Commons licence and your intended use is not permitted by statutory regulation or exceeds the permitted use, you will need to obtain permission directly from the copyright holder. To view a copy of this licence, visit http://creativecommons.org/licenses/by-nc/4.0/.

\section{References}

1. Chow CK, Teo KK, Rangarajan S, Islam S, Gupta R, Avezum A, et al. Prevalence, awareness, treatment, and control of hypertension in rural and urban communities in high-, middle-, and lowincome countries. JAMA. 2013;310:959-68.

2. Briasoulis A, Agarwal V, Tousoulis D, Stefanadis C. Effects of antihypertensive treatment in patients over 65 years of age: a meta-analysis of randomised controlled studies. Heart. 2014;100:317-23.

3. Beckett NS, Peters R, Fletcher AE, Staessen JA, Liu L, Dumitrascu D, et al. Treatment of hypertension in patients 80 years of age or older. N Engl J Med. 2008;358:1887-98.

4. Del Pinto R, Ferri C. Hypertension management at older age: an update. High Blood Press Cardiovasc Prev. 2019;26:27-36.

5. Corrao G, Rea F, Monzio Compagnoni M, Merlino L, Mancia G. Protective effects of antihypertensive treatment in patients aged 85 years or older. J Hypertens. 2017;35:1432-41.

6. Williams B, Mancia G, Spiering W, Agabiti Rosei E, Azizi M, Burnier M, et al. 2018 ESC/ESH Guidelines for the management of arterial hypertension. Eur Heart J. 2018;39:3021-104.

7. Whelton PK, Carey RM, Aronow WS, Casey DE Jr, Collins KJ, Dennison Himmelfarb C, et al. 2017 ACC/AHA/AAPA/ABC/ ACPM/AGS/APhA/ASH/ASPC/NMA/PCNA Guideline for the Prevention, Detection, Evaluation, and Management of High Blood Pressure in Adults: a Report of the American College of Cardiology/American Heart Association Task Force on Clinical Practice Guidelines. Hypertension. 2018;71:e13-115.

8. Banegas JR, López-García E, Dallongeville J, Guallar E, Halcox JP, Borghi C, et al. Achievement of treatment goals for primary prevention of cardiovascular disease in clinical practice across Europe: the EURIKA study. Eur Heart J. 2011;32:2143-52.

9. Wang YR, Alexander GC, Stafford RS. Outpatient hypertension treatment, treatment intensification, and control in Western Europe and the United States. Arch Intern Med. 2007;167:141-7.

10. Gale NK, Greenfield S, Gill P, Gutridge K, Marshall T. Patient and general practitioner attitudes to taking medication to prevent cardiovascular disease after receiving detailed information on risks and benefits of treatment: a qualitative study. BMC Fam Pract. 2011;12:59.

11. Krousel-Wood M, Joyce C, Holt E, Muntner P, Webber LS, Morisky DE, et al. Predictors of decline in medication adherence: results from the cohort study of medication adherence among older adults. Hypertension. 2011;58:804-10.

12. Corrao G, Parodi A, Zambon A, Heiman F, Filippi A, Cricelli $\mathrm{C}$, et al. Reduced discontinuation of antihypertensive treatment by two-drug combination as first step. Evidence from daily life practice. J Hypertens. 2010;28:1584-90.

13. Gupta AK, Arshad S, Poulter NR. Compliance, safety, and effectiveness of fixed-dose combinations of antihypertensive agents: a meta-analysis. Hypertension. 2010;55:399-407.

14. Volpe M, Rosei EA, Ambrosioni E, Cottone S, Cuspidi C, Borghi C, et al. 2012 consensus document of the Italian Society of Hypertension (SIIA): strategies to improve blood pressure control in Italy: from global cardiovascular risk stratification to combination therapy. High Blood Press Cardiovasc Prev. 2013;20:45-52.

15. Williams B, Mancia G, Spiering W, Agabiti Rosei E, Azizi M, Burnier M, et al. 2018 ESC/ESH Guidelines for the management of arterial hypertension: the Task Force for the management of arterial hypertension of the European Society of Cardiology and the European Society of Hypertension: The Task Force for the management of arterial hypertension of the European Society of Cardiology and the European Society of Hypertension. J Hypertens. 2018;36:1953-2041.

16. Weir MR, Hsueh WA, Nesbitt SD, Littlejohn TJ 3rd, Graff A, Shojaee A, et al. A titrate-to-goal study of switching patients uncontrolled on antihypertensive monotherapy to fixed-dose combinations of amlodipine and olmesartan medoxomil \pm hydrochlorothiazide. J Clin Hypertens. 2011;13:404-12.

17. Volpe M, Christian Rump L, Ammentorp B, Laeis P. Efficacy and safety of triple antihypertensive therapy with the olmesartan/ amlodipine/hydrochlorothiazide combination. Clin Drug Investig. 2012;32:649-64. 
18. Tocci G, Muiesan ML, Parati G, Agabiti Rosei E, Ferri C, Virdis A, et al. Trends in Prevalence, Awareness, Treatment, and Control of Blood Pressure Recorded From 2004 to 2014 During World Hypertension Day in Italy. J Clin Hypertens. 2016;18:551-6.

19. Torlasco C, Faini A, Makil E, Bilo G, Pengo M, Beaney T, et al. Nation-wide hypertension screening in Italy: data from May Measurements Month 2017-Europe. Eur Heart J Suppl. 2019;21:D66-70.

20. Beaney T, Burrell LM, Castillo RR, Charchar FJ, Cro S, Damasceno A, et al. May Measurement Month 2018: a pragmatic global screening campaign to raise awareness of blood pressure by the International Society of Hypertension. Eur Heart J. 2019;40:2006-17.

21. Choudhry NK, Shrank WH, Levin RL, Lee JL, Jan SA, Brookhart MA, et al. Measuring concurrent adherence to multiple related medications. Am J Manag Care. 2009;15:457-64.

22. DiMatteo MR, Giordani PJ, Lepper HS, Croghan TW. Patient adherence and medical treatment outcomes: a meta-analysis. Med Care. 2002;40:794-811.

23. Melgarejo JD, Maestre GE, Thijs L, Asayama K, Boggia J, Casiglia E, et al. Prevalence, Treatment, and Control Rates of Conventional and Ambulatory Hypertension Across 10 Populations in 3 Continents. Hypertension. 2017;70:50-8.

24. Rizzo ML. Statistical Computing with R, Second Edition. CRC Press; 2019. ISBN 9781466553323

25. Conn VS, Ruppar TM, Chase J-AD, Enriquez M, Cooper PS. Interventions to improve medication adherence in hypertensive patients: systematic review and meta-analysis. Curr Hypertens Rep. 2015;17:94.

26. Mizokami F, Koide Y, Noro T, Furuta K. Polypharmacy with common diseases in hospitalized elderly patients. Am J Geriatr Pharmacother. 2012;10:123-8.
27. Ferri C, Ferri L, Desideri G. Management of Hypertension in the Elderly and Frail Elderly. High Blood Press Cardiovasc Prev. 2017;24:1-11.

28. Webster R, Salam A, de Silva HA, Selak V, Stepien S, Rajapakse $\mathrm{S}$, et al. Fixed low-dose triple combination antihypertensive medication vs usual care for blood pressure control in patients with mild to moderate hypertension in sri lanka: a randomized clinical trial. JAMA. 2018;320:566-79.

29. Hermida RC, Ayala DE, Fernández JR, Mojón A, Smolensky MH. Hypertension: New perspective on its definition and clinical management by bedtime therapy substantially reduces cardiovascular disease risk. Eur J Clin Invest. 2018;48:e12909.

30. Holman H, Lorig K. Patients as partners in managing chronic disease. Partnership is a prerequisite for effective and efficient health care. BMJ. 2000;320:526-7.

31. Pietropaoli D, Del Pinto R, Ferri C, Wright JT Jr, Giannoni M, Ortu E, et al. Poor Oral Health and Blood Pressure Control Among US Hypertensive Adults: Results From the National Health and Nutrition Examination Survey 2009 to 2014. Hypertension. 2018;72:1365-73.

32. Del Pinto R, Pagliacci S, De Feo M, Grassi D, Ferri C. Italian Society of Hypertension and Federfarma .Prevalence of hypertension and associated cardiovascular risk factors among pharmacies customers: an Italian nationwide epidemiological survey. Eur J Prev Cardiol. 2020;27:1228-30.

33. Del Pinto R, Wright JT, Monaco A, Pietropaoli D, Ferri C. Vitamin $\mathrm{D}$ and blood pressure control among hypertensive adults: results from NHANES 2001-2014. J Hypertens. 2020;38:150-8.

34. Gupta P, Patel P, Horne R, Buchanan H, Williams B, Tomaszewski M. How to screen for non-adherence to antihypertensive therapy. Curr Hypertens Rep. 2016;18:89. 